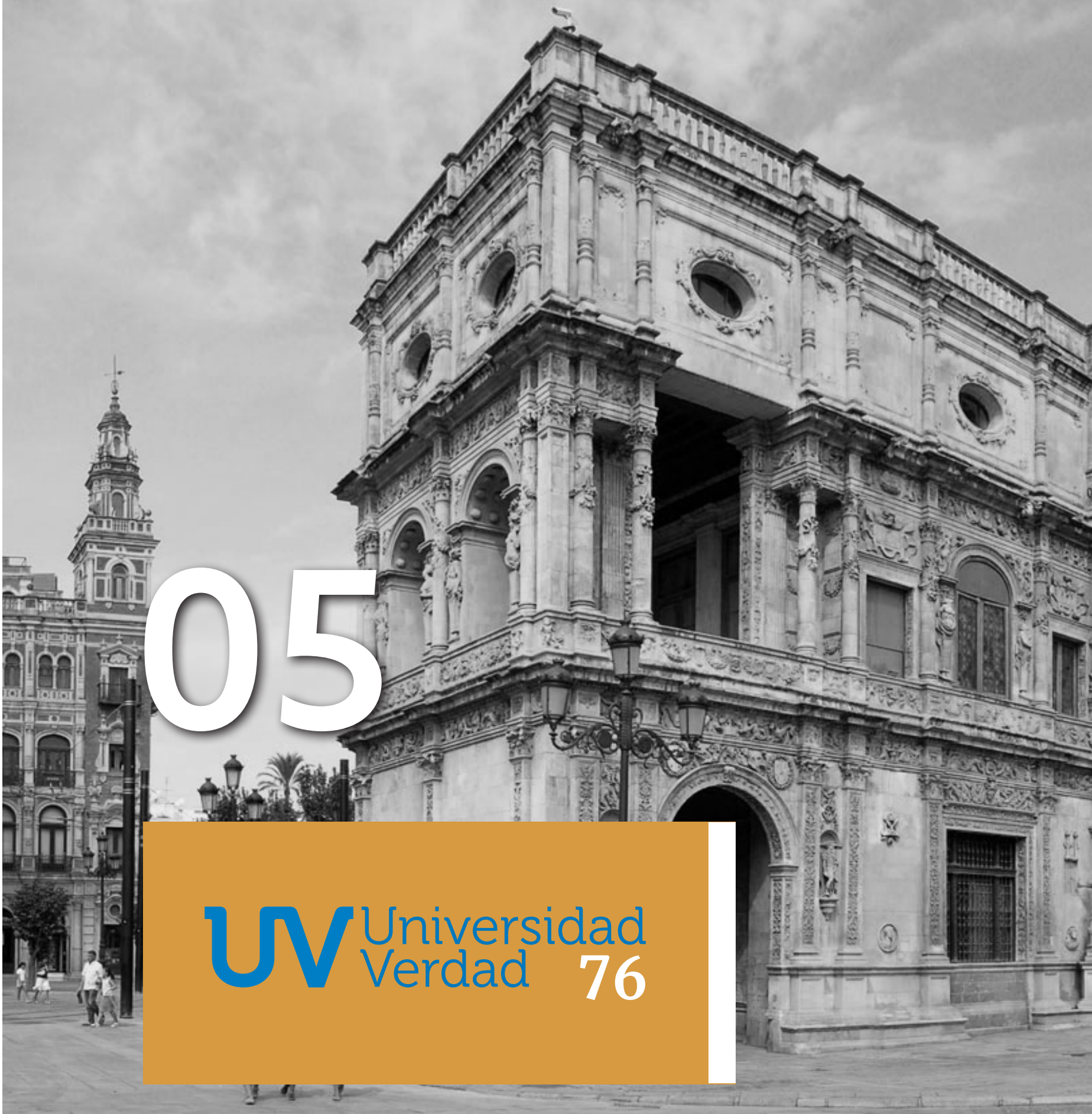

C.

Foto: Internet. http://www.uniquespain.travel/cosmopolita/es/top10/sevilla-visitas-rincones/ 


\section{COMUNICAR EL PATRIMONIO CULTURAL EN ENTORNOS DIGITALES: UNA COMPARATIVA DE ENFOQUES IBÉRICOS}

\section{Communicating cultural heritage in digital environments: a comparison of Iberian approaches}

Dr. Valeriano Piñeiro-Naval. Investigador postdoctoral en el LabCom - Comunicação e Artes. Universidade da Beira Interior. UBI. (Portugal) (vale.naval@labcom.ubi.pt) (http://orcid.org/0000-0001-9521-3364)

\section{Resumen}

En el actual contexto turístico y mediático, los destinos locales están obligados a seleccionar aquellos elementos de su patrimonio, ya sea material o inmaterial, que resulten más destacados o genuinos y que puedan actuar, no únicamente como una atracción turística, sino también como un factor identitario en el que la población autóctona se refleje. Así, el cometido fundamental del presente trabajo consiste en efectuar una instantánea de la comunicación del legado cultural en la Península Ibérica. De manera más específica, se llevó a cabo un análisis de contenido de una muestra representativa de 500 portales web municipales de España, y del universo poblacional de 308 sedes web locales de Portugal. Los resultados obtenidos indican que los elementos patrimoniales más destacados son las edificaciones religiosas, los eventos festivos y la gastronomía, encuadrados desde un punto de vista predominantemente local. Por otro lado, y tras las comparaciones efectuadas gracias al "Indicador de Información sobre Patrimonio Cultural", es posible afirmar que el discurso que se difunde en ambos países, a nivel digital, es muy similar no solo desde una comparativa ibérica, sino también desde una perspectiva interregional.

\section{Abstract}

In the current tourist and media context, local destinations are bound to select those elements of their heritage, whether material or immaterial, that are more prominent and genuine, and that can act not only as a tourist attraction, but also as an identity factor in that the local population is reflected. Thus, the fundamental purpose of this study was to carry out a picture of the communication of cultural legacy in the Iberian Peninsula. More specifically, a content analysis of a representative sample of 500 municipal websites in Spain and the universe of 308 local websites in Portugal has been carried out. The results indicate that the most outstanding heritage elements are religious buildings, festive events and gastronomy, framed from a predominantly local point of view. On the other hand, and after the comparisons based on the "Cultural Heritage Information Index", it is possible to affirm that the discourse disseminated in both countries on a digital level is very similar, and not only from an Iberian comparison, but also from an interregional perspective.

\section{Palabras clave}

Patrimonio cultural, Encuadre, Análisis de contenido, Sitios web, Municipios.

\section{Keywords}

Cultural heritage, Framing, Content analysis, Websites, Municipalities. 
1.

\section{Introducción y estado de la cuestión}

El propósito de este primer apartado consiste en delimitar unas coordenadas teóricas que actúen, en el mejor de los supuestos, como pautas para la interpretación - eso sí, un tanto superficial- de una serie de conceptos complejos y polisémicos. Se recogerán, para ello, determinadas definiciones formuladas a propósito de nociones como las de cultura, patrimonio, turismo o identidad, con el fin de ofrecer un marco conceptual que trate de organizar y estructurar una complicada realidad multidimensional: la comunicación del patrimonio cultural perteneciente a los destinos locales que se realiza mediante entornos digitales, y las consecuencias turísticas e implicaciones identitarias que esta difusión de la herencia popular pueda implicar.

Definir la noción de "cultura" es un cometido al cual numerosos y brillantes estudiosos han consagrado su obra. Tal podría ser el caso de Hofstade et al. (2010), quienes defienden que se trata de una programación mental colectiva, que distingue a los miembros de un grupo de individuos frente a los demás grupos. Esta idea concuerda a la perfección con la "Teoría de la Identidad Social" — en adelante, TIS - , cuyo principal exponente es Tajfel (1981) y que propugna que las categorías sociales se constituyen con arreglo a características compartidas que ejercen, a su vez, como rasgos comparativos y diferenciadores respecto de otros grupos; esto es, el "endogrupo" propio frente a los otros "exogrupos". Por su parte, Hanitzsch
(2007) asegura que la cultura es un compendio de valores, actitudes, prácticas y artefactos; es decir, una suma de elementos inherentes al ser humano y a su legado, tanto material como inmaterial.

Esta doble dimensión, la tangible y la intangible, es también prototípica del "patrimonio", que nos permite construir puentes entre el pasado y el presente, puesto que nos dota de una perspectiva histórica en un mundo sometido a constantes cambios (Bessière, 1998). Asimismo, el patrimonio consiste en un conglomerado de recursos que se consideran únicos, insustituibles, esenciales para la configuración de la identidad, tanto personal como colectiva, y necesarios para el fomento del respeto hacia lo propio y lo ajeno (Lowenthal, 2005).

Identificar y catalogar el patrimonio es una ardua tarea, de la cual se han responsabilizado, tradicionalmente, organismos internacionales como la UNESCO -United Nations Educational, Scientific and Cultural Organization - o ICOMOS - International Council on Monuments and Sites-. En líneas generales, y pese a que los elementos susceptibles de ser clasificados no siempre son atribuidos a departamentos estancos, el patrimonio se subdivide en natural y cultural y este, a su vez, en material - edificaciones de tipo religioso, civil y militar, museos, bibliotecas, teatros, etc.e inmaterial - gastronomía, tradiciones orales, artes del espectáculo, artesanía, etc.- (ICOMOS, 1999; UNESCO, 2003, 2005). Todos estos bienes muebles e inmuebles, así como las restantes manifestaciones de la cultura y la identidad populares, son uno de los principales reclamos de la industria turística, que representa un alto porcentaje del producto interior bruto mundial (World Tourism Organization, 2018).

En este sentido, el "turismo cultural", es decir, el movimiento de personas por razones estrictamente culturales (Galí-Espelt, 2012), es primordial en la estrategia nacional de algunos de los países más turísticos del mundo - véanse los casos de España, Francia o Italia, por citar los más representativos-. Y es que resulta innegable que el turismo y la cultura han estrechado cada vez más sus lazos, sobre todo cuando esta última adquiere un mayor protagonismo en la imagen y las estrategias de desarrollo regional (OECD, 2009). En el panorama actual, la diversificación de los gustos y preferencias, la fragmentación de la producción cultural y el acceso a las 
nuevas tecnologías y los medios digitales desafían a la producción monolítica de la cultura. Esta, además de un activo económico, también supone un mecanismo para configurar la identidad, un estímulo para la cohesión social y un acicate para la creatividad (Richards, 2014; Richards \& Marques, 2018; Sacco, 2011); factores con un impacto directo en la industria lúdica que propician, en buena medida, una modalidad denominada "turismo de distinción" (Cohen, 2005). El objetivo de esta práctica de ocio radica en la diferenciación de sus usuarios: individuos que poseen un grado relativamente alto de capital cultural, sofisticación y reflexividad con respecto a los activos patrimoniales que observan o con los que interactúan.

En lo que a la identidad se refiere, Castells (2006a) habla de la "identidad proyecto" como aquella que está sustentada en la autoidentificación, por parte de una colectividad humana, con narrativas y materiales culturales, históricos y territoriales. Estas narrativas y materiales se apoyan indiscutiblemente en el patrimonio, ya sea este un objeto, un monumento, una habilidad heredada o una representación simbólica, que, a su vez, debe ser considerado un rasgo de identidad distintivo de cualquier grupo social (Bessière, 2013). Tanto en el concepto de "identidad proyecto" como en la disyuntiva que propugna la TIS - basada en las comparaciones del "endogrupo" frente a los "exogrupos" - subyace la dialéctica entre lo propio y lo ajeno, o, en otras palabras, entre lo local y lo global. En este sentido, la voz "glocalización" surge como explicación a esa mezcla que, en términos culturales, se da entre los elementos locales y particulares con los mundializados. En opinión de Bolívar (2001), "es objeto de las ciencias sociales ver cómo las fuerzas globales interactúan con las culturas locales, al tiempo que son mediadas y filtradas por ellas" (p. 269). Dicha interacción resulta en una programación mental colectiva en la que confluyen elementos idiosincráticos, distintivos de cada grupo, con otros rasgos de carácter universal y, por tanto, uniformadores.

Son muchas las nociones que han germinado al amparo de las tendencias culturales e identitarias reproducidas desde finales del siglo XX: las consabidas "Globalización" (Featherstone, 1990), "McDonaldización" (Ritzer, 1992), "CocaColonización" (Urry, 1999) o "Glocalización" (Robertson, 2000); así como las más recientes "Multiculturalismo" (Bauman, 2013) o "Ikeaización" (Blackshaw, 2013), son apenas unos pocos ejemplos. Grosso modo, todas ellas sirven para referir las tensiones generadas a raíz del contacto entre las culturas locales o regionales con aquellas de envergadura global, fruto del desarrollo de los transportes, el turismo y las telecomunicaciones, que han convertido al mundo en un escenario nunca tan reducido en la historia de la humanidad.

Desde este planteamiento, cabe preguntarse: ¿Qué tipo de iniciativas emprenden los colectivos sociales para reaccionar contra estas dinámicas globales que uniformizan la cultura? Una de ellas consiste en impulsar un "discurso patrimonial autorizado" (Smith, 2006; Waterton \& Smith, 2010), que constituye una determinada representación de "nosotros mismos" y de "nuestro" paisaje, tradiciones y estilo de vida (Hallett \& Kaplan-Weinger, 2010). Este discurso construye una narrativa cimentada en la selección de aquellos atributos patrimoniales que son considerados, a juicio de los gestores culturales, como los más valiosos y apreciados. Así, recae sobre ellos una doble función: la turística, ya que atraen visitantes - exogrupos-; y la identitaria, en tanto que facilitan el auto-reconocimiento de la población local -endogrupo- - Este mecanismo de selección de determinados elementos de la realidad - enfatizados en los textos informativos en detrimento de los demás - es definitorio de la "Teoría del Encuadre" (Entman, 1993; Scheufele \& Tewksbury, 2007; Vreese, 2005), uno de los paradigmas conceptuales y metodológicos más extendidos en la investigación en ciencias de la comunicación (Borah, 2011; Bryant \& Miron, 2004; Matthes, 2009; Piñeiro-Naval \& Mangana, 2018, 2019; Saperas \& Carrasco-Campos, 2015; Vicente-Mariño \& López-Rabadán, 2009).

En este sentido, cabe subrayar la importancia de la comunicación en la relación que existe entre cultura, patrimonio y turismo (véase, por ejemplo: Piñeiro-Naval \& Morais, 2020), porque "si no se comunica la existencia del patrimonio cultural, este no existe para los públicos. Si no se comunica su identidad, no se conocen sus particularidades. Si no se comunica su diferencia, no hay diferencia" (Capriotti, 2008, p. 133). Por tanto, los destinos locales deberían, en primer lugar, inventariar aquellos elementos de su patrimonio material e inmaterial más destacados y genuinos, y que puedan actuar no únicamente como 
atracción turística, sino también como factor identitario en el que la población se refleje. El conocimiento del patrimonio de un determinado territorio y su exploración suponen la concreción de una experiencia turística distintiva y enriquecedora para todos los agentes implicados: desde los gestores hasta los visitantes, pasando por los autóctonos. De este modo, y en relativa medida, un discurso patrimonial debidamente elaborado mitigaría los efectos de unos flujos globales que tienden a diluir las naturales y necesarias diferencias entre unos pueblos y otros.

A modo de resumen, se presenta un esquema conceptual (Gráfico 1) que pretende reflejar algunas de las nociones anteriormente repasadas.

\section{2.}

\section{Materiales y método}

A tenor del marco conceptual brevemente expuesto en la introducción, el cometido fundamental del presente estudio radica en efectuar una radiografía de la comunicación del patrimonio cultural en la Península Ibérica. En detalle, los objetivos específicos del trabajo son los que siguen:
- OE1: Señalar los elementos del patrimonio cultural material e inmaterial más sobresalientes en los sitios web oficiales de los municipios de España y Portugal.

- $\mathrm{OE}_{2}$ : Identificar los encuadres dados a estos elementos patrimoniales, basados en la dialéctica identitaria existente entre lo local y lo global.

- $\mathrm{OE}_{3}$ : Efectuar comparaciones entre ambos países en lo concerniente a la difusión en línea de su patrimonio y enfoques.

La elección de los portales web de los municipios obedece a que estos son los organismos institucionales más próximos al ciudadano y, por ello, los que deben corresponder, en primera instancia, a sus demandas e intereses. En este sentido, cabe recordar que una gestión adecuada del patrimonio puede generar un mayor conocimiento y disfrute por parte del público local, además de la actividad turística asociada a su consumo experiencial por parte del público foráneo.

En lo que atañe a los sitios web, e inmersos de pleno en la denominada "sociedad red" (Castells, 2006b), es posible aseverar que estos han supuesto el primer servicio digital que brindaron las organizaciones públicas y privadas a los usuarios. Son, por lo tanto, una

\section{Gráfico 1}

Esquema conceptual de la investigación

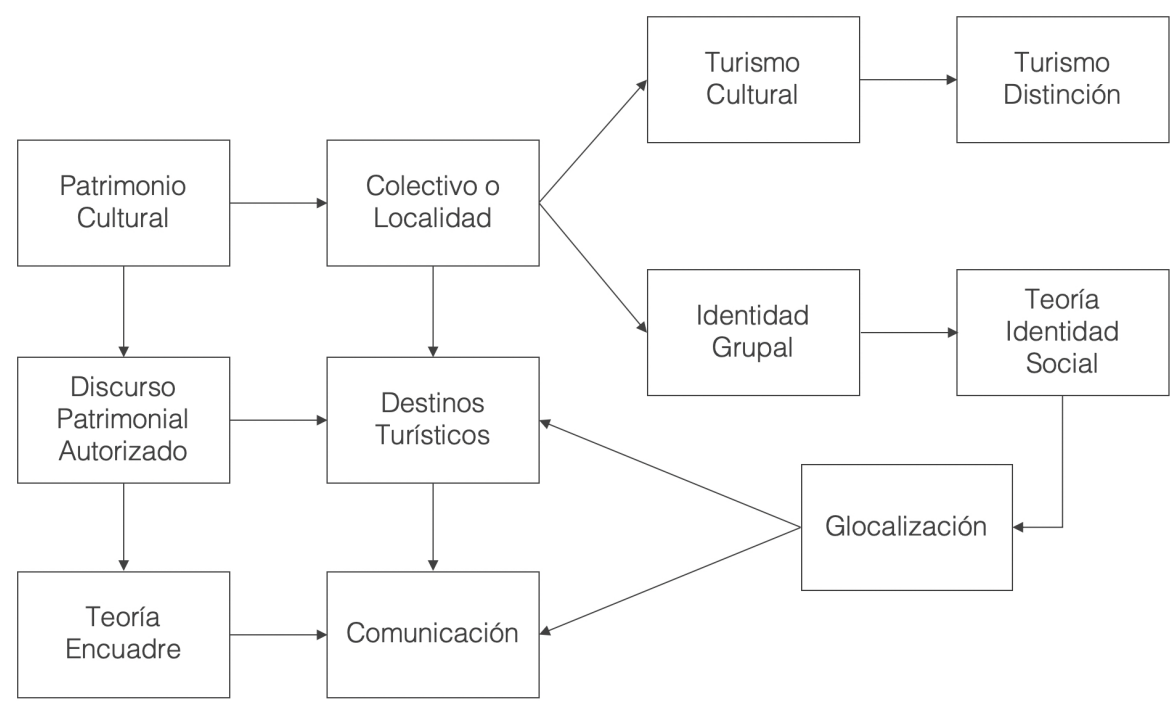




\section{Gráfico 2}

Ejemplos de unidades de análisis
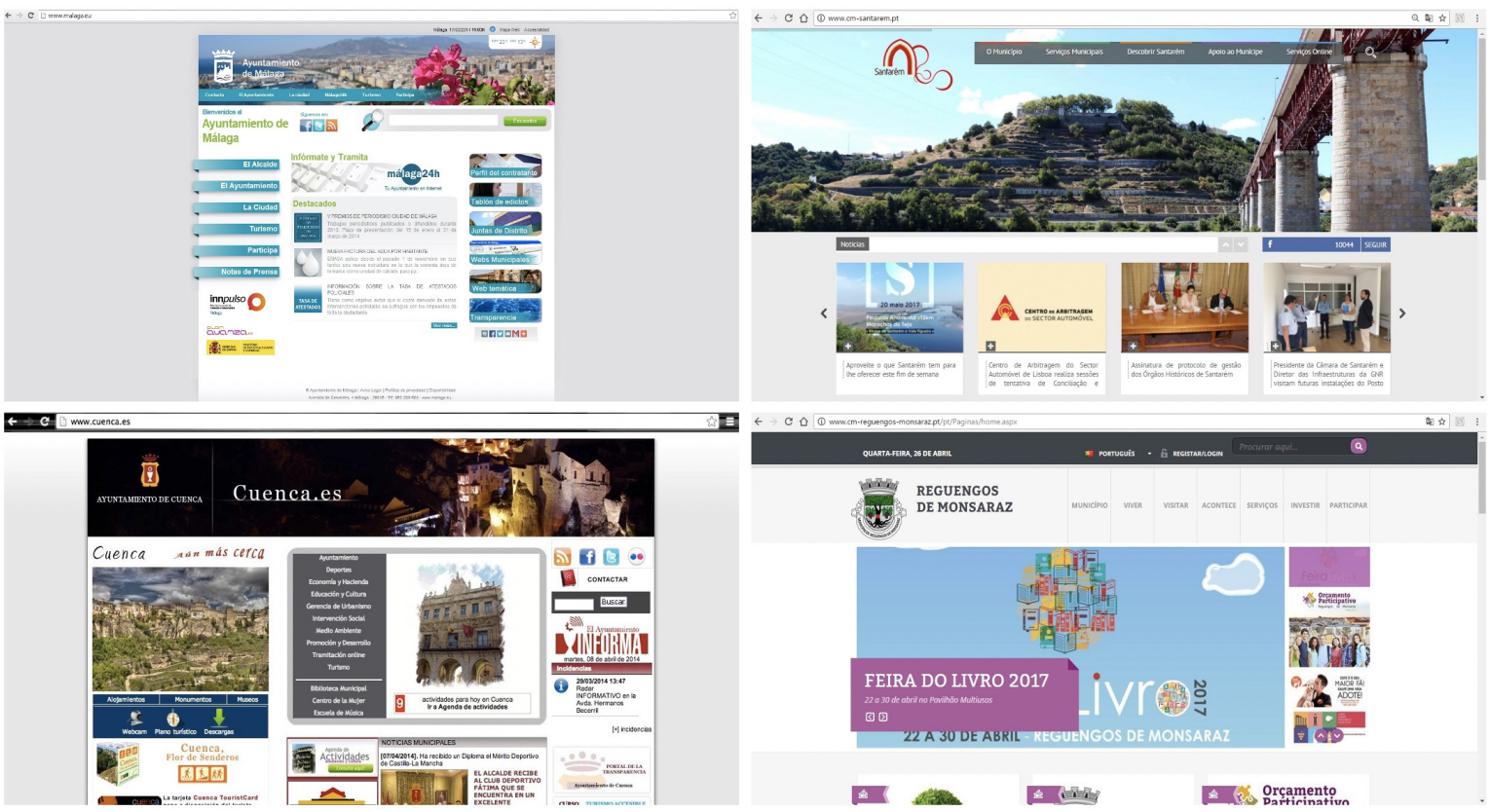

Nota. El gráfico muestra los sitios web de Málaga, Santarém, Cuenca y Reguengos de Monsaraz-de izquierda a derecha y de arriba abajo-.

suerte de "pioneros" en la propagación de las tecnologías de la información y la comunicación - TICen la arena mediática.

Desde el punto de vista metodológico, se empleó la técnica del "análisis de contenido" por ser sistemática, objetiva y cuantitativa (Wimmer \& Dominick, 2011); además de permeable a cualquier material de la comunicación humana (White \& Marsh, 2006), incluidos los contenidos web. Si bien es cierto que son necesarias algunas precauciones añadidas para su aplicación a ambientes interactivos (Piñeiro-Naval et al., 2018), uno de los puntos de partida para su ejecución consiste siempre en definir la muestra. En el caso de España, la estrategia de muestreo fue "polietápica" (Neuendorf, 2017), ya que se produjo en distintas fases: se estratificaron los municipios según las 50 provincias del país, se seleccionó un caso crítico de cada una - el sitio web de la capital - , y se sumaron 9 casos más por provincia de forma aleatoria. De este modo, se llegó a un total de $\mathrm{n}=500$ portales web, que acarrean un margen de error de $\sim 4.2 \%$, con respecto a las 8131 localidades del país.
En cuanto al caso portugués, fue analizado el universo: $\mathrm{n}=308$ sedes web municipales.

Tras el muestreo, "se debe elaborar un libro de códigos - codebook - en el que se especifique, con gran detalle, cómo debe evaluarse cada criterio o variable" (Igartua, 2006, p. 204). Esta guía para la codificación del corpus de análisis, empleada en estudios previos (Piñeiro-Naval \& Igartua, 2012; Piñeiro-Naval, Igartua \& Rodríguez-de-Dios, 2018; Piñeiro-Naval \& Serra, 2019), estuvo compuesta por estas variables (Tabla 1): 
Tabla 1

Variables del estudio

\begin{tabular}{|c|c|c|c|c|}
\hline \multirow[t]{2}{*}{$\mathbf{N}^{\mathbf{o}}$} & \multirow[t]{2}{*}{ Variables } & \multirow[t]{2}{*}{ Categorías } & \multicolumn{2}{|c|}{ Fiabilidad $\left(\alpha_{k}\right)$} \\
\hline & & & España & Portugal \\
\hline \multicolumn{5}{|c|}{ Elementos del Patrimonio Cultural Material } \\
\hline 1. & $\begin{array}{l}\text { Bibliotecas, filmotecas u otros } \\
\text { archivos documentales }\end{array}$ & $\begin{array}{l}\text { 0=no existe información, } \\
\text { 1=sí existe información. }\end{array}$ & 0.60 & 0.80 \\
\hline 2. & Enfoque identitario bibliotecas & $\begin{array}{l}0=\text { no se informa, } 1=\text { local, } 2=\text { regional, } 3=\text { na }- \\
\text { cional, } 4=\text { global, } 9=\text { ningún enfoque. }\end{array}$ & 0.50 & 0.48 \\
\hline 3. & Catedrales o basílicas & $\begin{array}{l}\text { 0=no existe información, } \\
1=\text { sí existe información. }\end{array}$ & 0.92 & 1 \\
\hline 4. & Enfoque identitario catedrales & $\begin{array}{l}\text { 0=no se informa, 1=local, } 2=\text { regional, } 3=\text { na- } \\
\text { cional, } 4=\text { global, 9=ningún enfoque. }\end{array}$ & 0.85 & 1 \\
\hline 5. & Conjuntos o centros urbanos & $\begin{array}{l}0=\text { no existe información, } \\
\text { 1=sí existe información. }\end{array}$ & 0.51 & 0.81 \\
\hline 6. & Enfoque identitario conjuntos & $\begin{array}{l}\text { 0=no se informa, } 1=\text { local, } 2=\text { regional, } 3=\text { na }- \\
\text { cional, } 4=\text { global, 9=ningún enfoque. }\end{array}$ & 0.47 & 0.75 \\
\hline 7. & $\begin{array}{l}\text { Edificaciones civiles o militares: } \\
\text { casonas, castillos, murallas o puentes }\end{array}$ & $\begin{array}{l}\text { 0=no existe información, } \\
\text { 1=sí existe información. }\end{array}$ & 0.72 & 0.75 \\
\hline 8. & $\begin{array}{l}\text { Enfoque identitario edificaciones } \\
\text { civiles o militares }\end{array}$ & $\begin{array}{l}\text { 0=no se informa, 1=local, 2=regional, 3=na- } \\
\text { cional, 4=global, 9=ningún enfoque. }\end{array}$ & 0.60 & 0.66 \\
\hline 9. & $\begin{array}{l}\text { Edificaciones religiosas: iglesias, } \\
\text { capillas, ermitas o monasterios }\end{array}$ & $\begin{array}{l}\text { 0=no existe información, } \\
\text { 1=sí existe información. }\end{array}$ & 0.88 & 0.89 \\
\hline 10. & $\begin{array}{l}\text { Enfoque identitario edificaciones } \\
\text { religiosas }\end{array}$ & $\begin{array}{l}\text { 0=no se informa, 1=local, 2=regional, 3=na- } \\
\text { cional, 4=global, 9=ningún enfoque. }\end{array}$ & 0.67 & 0.89 \\
\hline 11. & $\begin{array}{l}\text { Estatuas o esculturas que simbolicen } \\
\text { a personajes emblemáticos }\end{array}$ & $\begin{array}{l}\text { 0=no existe información, } \\
\text { 1=sí existe información. }\end{array}$ & 0.75 & 0.84 \\
\hline 12. & $\begin{array}{l}\text { Enfoque identitario estatuas o } \\
\text { esculturas }\end{array}$ & $\begin{array}{l}\text { 0=no se informa, 1=local, 2=regional, 3=na- } \\
\text { cional, } 4=\text { global, 9=ningún enfoque. }\end{array}$ & 0.73 & 0.83 \\
\hline 13. & $\begin{array}{l}\text { Museos, salas de exposiciones o casas } \\
\text { de cultura }\end{array}$ & $\begin{array}{l}\text { 0=no existe información, } \\
\text { 1=sí existe información. }\end{array}$ & 0.75 & 0.64 \\
\hline 14. & Enfoque identitario museos & $\begin{array}{l}\text { 0=no se informa, 1=local, } 2=\text { regional, 3=na- } \\
\text { cional, 4=global, 9=ningún enfoque. }\end{array}$ & 0.56 & 0.67 \\
\hline 15. & $\begin{array}{l}\text { Parques o jardines de especial interés } \\
\text { arquitectónico }\end{array}$ & $\begin{array}{l}\text { 0=no existe información, } \\
\text { 1=sí existe información. }\end{array}$ & 0.78 & 0.71 \\
\hline 16. & $\begin{array}{l}\text { Enfoque identitario parques o } \\
\text { jardines }\end{array}$ & $\begin{array}{l}0=\text { no se informa, 1=local, } 2=\text { regional, } 3=\text { na- } \\
\text { cional, 4=global, 9=ningún enfoque. }\end{array}$ & 0.56 & 0.74 \\
\hline
\end{tabular}




\begin{tabular}{|c|c|c|c|c|}
\hline 17. & Plazas de toros & $\begin{array}{l}0=\text { no existe información, } \\
1=\text { sí existe información. }\end{array}$ & 0.91 & 0.66 \\
\hline 18. & Enfoque identitario plazas de toros & $\begin{array}{l}\text { O=no se informa, 1=local, 2=regional, 3=na- } \\
\text { cional, 4=global, 9=ningún enfoque. }\end{array}$ & 0.86 & 0.68 \\
\hline 19. & $\begin{array}{l}\text { Plazas o patios de especial interés } \\
\text { arquitectónico }\end{array}$ & $\begin{array}{l}0=\text { no existe información, } \\
1=\text { sí existe información. }\end{array}$ & 0.65 & 0.73 \\
\hline 20. & Enfoque identitario plazas o patios & $\begin{array}{l}\text { O=no se informa, } 1=\text { local, } 2=\text { =regional, } 3=\text { na- } \\
\text { cional, } 4=\text { global, } 9=\text { ningún enfoque. }\end{array}$ & 0.54 & 0.74 \\
\hline 21. & Restos o depósitos arqueológicos & $\begin{array}{l}\text { 0=no existe información, } \\
1=\text { sí existe información. }\end{array}$ & 0.50 & 0.88 \\
\hline 22. & $\begin{array}{l}\text { Enfoque identitario restos } \\
\text { arqueológicos }\end{array}$ & $\begin{array}{l}0=\text { no se informa, } 1=\text { local, } 2=\text { regional, } 3=\text { na- } \\
\text { cional, } 4=\text { global, } 9=\text { ningún enfoque. }\end{array}$ & 0.50 & 0.80 \\
\hline 23. & Teatros o anfiteatros & $\begin{array}{l}\text { 0=no existe información, } \\
1=\text { sí existe información. }\end{array}$ & 0.73 & 0.63 \\
\hline 24. & $\begin{array}{l}\text { Enfoque identitario teatros o } \\
\text { anfiteatros }\end{array}$ & $\begin{array}{l}\text { 0=no se informa, 1=local, 2=regional, 3=na- } \\
\text { cional, 4=global, 9=ningún enfoque. }\end{array}$ & 0.58 & 0.58 \\
\hline \multicolumn{5}{|c|}{ Elementos del Patrimonio Cultural Inmaterial } \\
\hline 25. & Artesanía tradicional & $\begin{array}{l}\text { 0=no existe información, } \\
\text { 1=sí existe información. }\end{array}$ & 0.71 & 0.89 \\
\hline 26. & Enfoque identitario artesanía & $\begin{array}{l}\text { 0=no se informa, 1=local, 2=regional, 3=na- } \\
\text { cional, 4=global, 9=ningún enfoque. }\end{array}$ & 0.56 & 0.90 \\
\hline 27. & $\begin{array}{l}\text { Artes del espectáculo: festivales, } \\
\text { folklore, danza, flamenco o teatro }\end{array}$ & $\begin{array}{l}0=\text { no existe información, } \\
1=\text { sí existe información. }\end{array}$ & 0.63 & 0.54 \\
\hline 28. & $\begin{array}{l}\text { Enfoque identitario artes del } \\
\text { espectáculo }\end{array}$ & $\begin{array}{l}0=\text { no se informa, 1=local, } 2=\text { regional, 3=na- } \\
\text { cional, } 4=\text { global, 9=ningún enfoque. }\end{array}$ & 0.61 & 0.47 \\
\hline 29. & Eventos festivos municipales & $\begin{array}{l}0=\text { no existe información, } \\
1=\text { sí existe información. }\end{array}$ & 0.72 & 0.78 \\
\hline 30. & Enfoque identitario eventos festivos & $\begin{array}{l}\text { 0=no se informa, 1=local, 2=regional, 3=na- } \\
\text { cional, 4=global, 9=ningún enfoque. }\end{array}$ & 0.63 & 0.66 \\
\hline 31. & Eventos religiosos y eclesiásticos & $\begin{array}{l}\text { 0=no existe información, } \\
1=\text { sí existe información. }\end{array}$ & 0.63 & 0.73 \\
\hline 32. & $\begin{array}{l}\text { Enfoque identitario eventos religio- } \\
\text { sos }\end{array}$ & $\begin{array}{l}\text { 0=no se informa, 1=local, 2=regional, 3=na- } \\
\text { cional, 4=global, 9=ningún enfoque. }\end{array}$ & 0.53 & 0.68 \\
\hline 33. & Festejos ligados a la tauromaquia & $\begin{array}{l}\text { 0=no existe información, } \\
1=\text { sí existe información. }\end{array}$ & 0.70 & 0.84 \\
\hline 34. & Enfoque identitario tauromaquia & $\begin{array}{l}\text { 0=no se informa, 1=local, 2=regional, 3=na- } \\
\text { cional, 4=global, 9=ningún enfoque. }\end{array}$ & 0.61 & 0.82 \\
\hline 35. & $\begin{array}{l}\text { Figuras destacadas, originarias o } \\
\text { características del municipio }\end{array}$ & $\begin{array}{l}0=\text { no existe información, } \\
1=\text { sí existe información. }\end{array}$ & 0.76 & 0.65 \\
\hline
\end{tabular}




\begin{tabular}{|c|c|c|c|c|}
\hline 36. & Enfoque identitario figuras & $\begin{array}{l}0=\text { no se informa, } 1=\text { local, } 2=\text { regional, } 3=\text { na- } \\
\text { cional, } 4=\text { global, } 9=\text { ningún enfoque. }\end{array}$ & 0.68 & 0.57 \\
\hline 37. & Gastronomía típica del lugar & $\begin{array}{l}\text { 0=no existe información, } \\
\text { 1=sí existe información. }\end{array}$ & 0.77 & 0.80 \\
\hline 38. & Enfoque identitario gastronomía & $\begin{array}{l}0=\text { no se informa, } 1=\text { local, } 2=\text { regional, } 3=\text { na- } \\
\text { cional, } 4=\text { global, } 9=\text { ningún enfoque. }\end{array}$ & 0.55 & 0.54 \\
\hline 39. & $\begin{array}{l}\text { Tradiciones o expresiones orales: } \\
\text { canciones, cuentos, leyendas o } \\
\text { himnos }\end{array}$ & $\begin{array}{l}0=\text { no existe información, } \\
\text { 1=sí existe información. }\end{array}$ & 0.63 & 0.80 \\
\hline 40. & Enfoque identitario tradiciones orales & $\begin{array}{l}0=\text { no se informa, } 1=\text { local, } 2=\text { regional, 3=na- } \\
\text { cional, 4=global, 9=ningún enfoque. }\end{array}$ & 0.51 & 0.83 \\
\hline \multicolumn{5}{|c|}{ Posibles ubicaciones de la información patrimonial } \\
\hline 41. & Sitio web institucional & $\begin{array}{l}0=\text { no se ubica la información, } \\
1=\text { sí se ubica información. }\end{array}$ & 1 & 1 \\
\hline 42. & Sitio web temático sobre patrimonio & $\begin{array}{l}\text { 0=no se ubica información, } \\
\text { 1=sí se ubica información. }\end{array}$ & 0.87 & 0.65 \\
\hline & & Fiabilidad Media Total $\left(\alpha_{\mathrm{k}}\right)$ : & 0.67 & 0.75 \\
\hline
\end{tabular}

En total, el libro de códigos agrega 42 ítems acerca de los elementos del patrimonio cultural, tanto material -12 ítems dicotómicos o dummy-, con sus consiguientes enfoques -12 variables ordinales-, como inmaterial -8 ítems dummy-, con sus respectivos encuadres -8 variables ordinales-; sin olvidar, además, las dos variables dedicadas a la presencia de la información patrimonial, ya fuese en el sitio web oficial o en cualquier otro de carácter temático, enlazado al principal y destinado a la difusión de los atractivos culturales de la localidad — ambas variables dummy-.

Es clave puntualizar que la recolección de datos se dio en dos lapsos temporales bien diferenciados: en el caso español, la codificación transcurrió desde el 17 de febrero hasta el 14 de abril de 2014; mientras que los portales web de los municipios portugueses fueron inspeccionados desde el 16 de marzo hasta el 8 de junio de 2017. Este hecho hace que las comparaciones deban ser efectuadas con cierta cautela. Por otro lado, en la recolección de los datos españoles participaron 4 codificadores, y en el caso portugués intervinieron apenas 2 analistas.
En aras de chequear la fiabilidad del proceso, y debido a la multiplicidad de parámetros estadísticos existentes - un total de 22, según Zhao, Liu \& Deng (2013) - , se optó por seguir la recomendación de Hayes y Krippendorff (2007), quienes aseguran que el "Alpha de Krippendorff" es el más adecuado ya que "se generaliza a través de escalas de medición, se puede utilizar con cualquier número de observadores, con o sin los datos que falten, y satisface todos los criterios importantes para una buena medida de la fiabilidad" (p. 78). Así pues, los valores promedio del Alpha para el total de las 42 variables - detallados en la Tabla $1-$ son los siguientes: $\alpha_{k}=0.67$ para la codificación de los sitios web españoles, y $a_{k}=0.75$ para los portugueses, unos datos aceptables (Krippendorff, 2011).

\subsection{Indicador de información sobre patrimonio cultural}

Antes de proceder con el reporte de los resultados, es necesario aludir al "Indicador de Información sobre Patrimonio Cultural" - a partir de ahora, I2PC-, pues gracias a él fueron efectuadas algunas de las comparaciones que, a continuación, se mostrarán. 
La expresión alfanumérica que condensa la composición del índice es:

- $\mathrm{I} 2 \mathrm{PC}=\Sigma\left(\mathrm{x}_{1}, \mathrm{x}_{2}, \mathrm{x}_{3} \ldots \mathrm{x}_{\mathrm{n}}\right) / \mathrm{n}$; donde $\mathrm{n}=20$ variables dummy que aluden al patrimonio cultural, tanto tangible como intangible - véase Tabla 1-.

Como todos estos ítems son dicotómicos ( $0=$ ausencia de información, 1 = presencia de información), el indicador agregado resultante, cuyo poder explicativo es muy superior al de cada una de las variables por separado, también se rige por un rango que va de 0 a 1. Su consistencia interna hubo de calcularse para los dos casos: $\alpha_{c}=0.79$ para España, $\mathrm{y} \alpha_{c}=0.60$ para Portugal. Nuevamente, son valores correctos teniendo en cuenta el carácter exploratorio del trabajo (Hair et al., 2010).

\section{Tabla 2}

Presencia de elementos patrimoniales y sus enfoques según país (\%)

\begin{tabular}{|c|c|c|c|c|c|c|c|c|}
\hline \multirow{2}{*}{$\mathrm{N}^{\mathrm{o}}$} & \multirow{2}{*}{$\begin{array}{c}\text { Elementos } \\
\text { Patrimoniales }\end{array}$} & \multirow{2}{*}{ País } & \multicolumn{6}{|c|}{ Enfoques Identitarios } \\
\hline & & & Sin Enfoque & Local & Regional & Nacional & Global & Total \\
\hline \multicolumn{9}{|c|}{ Elementos del Patrimonio Cultural Material } \\
\hline \multirow{2}{*}{1.} & \multirow{2}{*}{ Bibliotecas } & España & 15.2 & 51.8 & 5.2 & 0.8 & 0.4 & 73.4 \\
\hline & & Portugal & 30.2 & 38.3 & 0.6 & 11.4 & 0.6 & 81.1 \\
\hline \multirow[t]{2}{*}{2.} & \multirow{2}{*}{ Catedrales } & España & 4.2 & 2.6 & 1.2 & 3.2 & 2 & 13.2 \\
\hline & & Portugal & 1.3 & 0.3 & - & 3.2 & 0.6 & 5.4 \\
\hline \multirow[t]{2}{*}{3.} & \multirow{2}{*}{$\begin{array}{l}\text { Conjuntos } \\
\text { urbanos }\end{array}$} & España & 13.8 & 17.2 & 2 & 4.6 & 1.6 & 39.2 \\
\hline & & Portugal & 15.3 & 4.2 & 2.6 & 7.1 & 2.9 & 32.1 \\
\hline \multirow[t]{2}{*}{4.} & \multirow{2}{*}{$\begin{array}{l}\text { Edificaciones } \\
\text { civiles }\end{array}$} & España & 27.4 & 28.8 & 8 & 10.4 & 4.2 & 78.8 \\
\hline & & Portugal & 35.7 & 17.5 & 5.2 & 23.4 & 3.6 & 85.4 \\
\hline \multirow[t]{2}{*}{5.} & \multirow{2}{*}{$\begin{array}{l}\text { Edificaciones } \\
\text { religiosas }\end{array}$} & España & 34.4 & 32.2 & 8.6 & 10.2 & 1.8 & 87.2 \\
\hline & & Portugal & 38.3 & 19.5 & 5.2 & 21.1 & 3.2 & 87.3 \\
\hline \multirow{2}{*}{6.} & \multirow{2}{*}{ Estatuas } & España & 10.4 & 10.4 & 2.6 & 1.2 & 0.8 & 25.4 \\
\hline & & Portugal & 29.5 & 11.4 & 1.6 & 6.5 & 1 & 50 \\
\hline \multirow{2}{*}{7.} & \multirow{2}{*}{ Museos } & España & 25.8 & 23.6 & 8.8 & 4.6 & 8 & 71 \\
\hline & & Portugal & 33.1 & 27.3 & 12.3 & 9.7 & 3.9 & 86.3 \\
\hline \multirow{2}{*}{8.} & \multirow{2}{*}{ Parques } & España & 19.8 & 12.4 & 2.2 & 1 & 2.2 & 37.6 \\
\hline & & Portugal & 35.1 & 6.5 & 0.3 & 1 & 1.6 & 44.5 \\
\hline \multirow{2}{*}{9.} & \multirow{2}{*}{ Plazas de toros } & España & 5 & 3.8 & 0.6 & 1.2 & 0.6 & 11.2 \\
\hline & & Portugal & 2.9 & 1.6 & - & 1 & - & 5.5 \\
\hline
\end{tabular}

Por último, y con la intención de efectuar algunas comparaciones entre regiones del mismo país y correlacionar la población de los municipios con su nivel de información patrimonial, se tomaron los datos geográficos y demográficos correspondientes a cada localidad de los Institutos Nacionales de Estadística de España y Portugal, para poder triangularlos (Denzin, 2012), así, con los valores del I2PC.

\section{3.}

\section{Resultados}

La Tabla 2 recoge, en primera instancia, todos los elementos del patrimonio, y sus respectivos enfoques, para cada país: 


\begin{tabular}{|c|c|c|c|c|c|c|c|c|}
\hline \multirow{2}{*}{10.} & \multirow{2}{*}{ Plazas o patios } & España & 26.5 & 18.4 & 1.2 & 1.4 & 0.8 & 47.4 \\
\hline & & Portugal & 19.2 & 2.3 & - & 1 & - & 22.5 \\
\hline \multirow{2}{*}{11.} & \multirow{2}{*}{$\begin{array}{l}\text { Restos } \\
\text { arqueológicos }\end{array}$} & España & 17.8 & 10 & 6 & 6.6 & 3.4 & 43.8 \\
\hline & & Portugal & 26.6 & 8.4 & 4.5 & 10.1 & 3.6 & 53.2 \\
\hline \multirow{2}{*}{12.} & \multirow{2}{*}{ Teatros } & España & 11.4 & 11 & 2 & 2.4 & 0.8 & 27.6 \\
\hline & & Portugal & 24.4 & 11.7 & 2.3 & 2.6 & 0.6 & 41.6 \\
\hline \multicolumn{9}{|c|}{ Elementos del Patrimonio Cultural Inmaterial } \\
\hline \multirow{2}{*}{13.} & \multirow{2}{*}{ Artesanía } & España & 6 & 10.8 & 2.8 & 2.4 & 1.6 & 23.6 \\
\hline & & Portugal & 26 & 20.1 & 6.2 & 2.9 & 2.9 & 58.1 \\
\hline \multirow{2}{*}{14.} & \multirow{2}{*}{$\begin{array}{l}\text { Artes del } \\
\text { espectáculo }\end{array}$} & España & 9.6 & 9.2 & 4.8 & 3.2 & 12.8 & 39.6 \\
\hline & & Portugal & 14 & 6.2 & 4.5 & 3.6 & 9.4 & 37.7 \\
\hline \multirow{2}{*}{15.} & \multirow{2}{*}{$\begin{array}{l}\text { Eventos } \\
\text { festivos }\end{array}$} & España & 27.6 & 39.4 & 9.8 & 7.4 & 4 & 88.2 \\
\hline & & Portugal & 47.4 & 11 & 2.6 & 3.2 & 1.9 & 66.1 \\
\hline \multirow{2}{*}{16.} & \multirow{2}{*}{$\begin{array}{l}\text { Eventos } \\
\text { religiosos }\end{array}$} & España & 25.6 & 34 & 8.6 & 5.6 & 3.4 & 77.2 \\
\hline & & Portugal & 49.7 & 6.5 & 1.6 & 1.3 & 7.8 & 66.9 \\
\hline \multirow{2}{*}{17.} & \multirow{2}{*}{$\begin{array}{l}\text { Festejos } \\
\text { tauromáquicos }\end{array}$} & España & 9 & 5.2 & 1.2 & 1.8 & 1.2 & 18.4 \\
\hline & & Portugal & 2.9 & 2.3 & 0.3 & 1.3 & 0.3 & 7.1 \\
\hline \multirow{2}{*}{18.} & \multirow{2}{*}{ Figuras } & España & 5.8 & 7 & 4.2 & 4.6 & 11.8 & 33.4 \\
\hline & & Portugal & 5.8 & 2.6 & 0.6 & 12.3 & 2.9 & 24.2 \\
\hline \multirow{2}{*}{19.} & \multirow{2}{*}{ Gastronomía } & España & 18.2 & 22.6 & 15.6 & 4.8 & 8 & 69.2 \\
\hline & & Portugal & 22.7 & 24.4 & 31.5 & 8.4 & 4.5 & 91.5 \\
\hline \multirow{2}{*}{20.} & \multirow{2}{*}{$\begin{array}{l}\text { Tradiciones } \\
\text { orales }\end{array}$} & España & 6.6 & 19.4 & 2.2 & 1.4 & 2.2 & 31.8 \\
\hline & & Portugal & 5.8 & 7.5 & 2.3 & 2.6 & 1.6 & 19.8 \\
\hline
\end{tabular}

En el ámbito de lo material, el elemento más sobresaliente tiene que ver con las edificaciones de tipo religioso, prácticamente idéntico en ambos países (87.2\% y $87.3 \%)$. En lo concerniente al patrimonio inmaterial, en España destacan los eventos festivos municipales (88.2\%), mientras que en Portugal es la gastronomía (91.5\%) la que abandera, sin discusión, las expresiones intangibles de su herencia popular. En cuanto al tratamiento identitario, prima la información donde no se enfatiza ningún encuadre específico. Entre los demás, el local es el que más suele abundar en los dos países, seguido del regional en España y del nacional en Portugal.

Para dar inicio a las comparaciones, cabe observar, antes de nada, los datos del I2PC de cada país: $M_{\text {I2PC-Esp }}=0.469(D T=0.193), M_{\text {I2PC-Por }}=0.468$ $(D T=0.136)$. Son medias prácticamente idénticas que no exhiben, por ende, diferencias estadísticamente significativas $[t(806)=1.099, p=0.864, d=$ 0.005]. Así pues, tanto España como Portugal llevan a cabo esfuerzos equiparables en lo que respecta a la divulgación de sus atractivos patrimoniales a nivel municipal. Si ahondamos ahora en las comparaciones inherentes a cada país, resulta interesante saber si existen diferencias entre el litoral y el interior (Gráfico 3): 


\section{Gráfico 3}

Comparaciones geográficas interregionales en los países

$$
\square \text { Litoral } \square \text { Interior }
$$

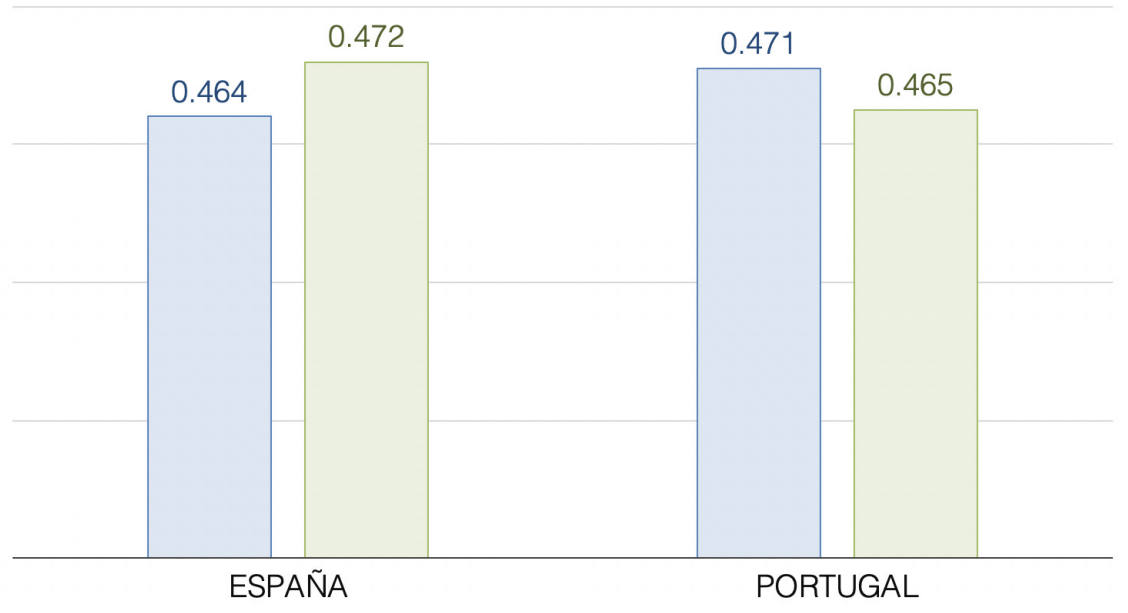

Tanto en España $[t(498)=-0.473, p=0.637, d=$ -0.041] como en Portugal [ $t(306)=0.328, p=0.743$, $d=0.044]$, no existen diferencias estadísticamente significativas entre los municipios del litoral y del interior, en lo referente a la información patrimonial de sus sitios web. Donde sí se producen datos estadísticamente significativos es en las correlaciones entre el I2PC y la población de las localidades: $r$ $(498)=0.298, p<0.001$ para los sitios de España, $\mathrm{y}$ $r(306)=0.232, p<0.001$ para Portugal. Este hecho manifiesta que, a medida que las ciudades aumentan su tamaño, la información patrimonial contenida en sus portales web también se incrementa.

Para finalizar, se midió la ubicación de los elementos patrimoniales, diferenciando entre los sitios web oficiales y posibles sitios temáticos enlazados al principal, cuyo propósito fuese la difusión del legado cultural de los pueblos. Pues bien, en la Tabla 3 puede observarse la distribución entre las posibles ubicaciones: 


\section{Tabla 3}

Asociación entre las ubicaciones de la información (\% columna)

\begin{tabular}{|c|c|c|c|}
\hline \multirow{2}{*}{ Otro sitio temático } & $\begin{array}{c}\text { \% } \\
\text { Total }\end{array}$ & $\begin{array}{c}\text { Sí se ubica la } \\
\text { información }\end{array}$ & $\begin{array}{c}\text { No se ubica la } \\
\text { información }\end{array}$ \\
\hline \multicolumn{4}{|c|}{ España } \\
\hline Sí se ubica la información & 30.2 & $29.6-$ & $83.3+$ \\
No se ubica la información & 69.8 & $70.4^{+}$ & $16.7-$ \\
\hline$N$ & 500 & 494 & 6 \\
\hline \multicolumn{4}{|c|}{ Portugal } \\
\hline Sí se ubica la información & 25.6 & $25.2-$ & $100+$ \\
No se ubica la información & 74.4 & $74.8+$ & $0-$ \\
\hline$N$ & 308 & 306 & 2 \\
\hline
\end{tabular}

Nota. - Valor estadísticamente menor (análisis de los residuos tipificados corregidos) + Valor estadísticamente mayor (análisis de los residuos tipificados corregidos)

Tanto para España $\left[X^{2}(1, N=500)=8.133, p<0.004\right.$, $v=0.128]$, como para Portugal $\left[X^{2}(1, N=308)=5.83\right.$, $p<0.05, v=0.016]$ los datos son estadísticamente relevantes. Si bien es cierto que en ambos países la mayor parte de la información está localizada en los sitios web oficiales, ambos poseen también una elevada proporción de municipios (29.6\% y $25.2 \%$, respectivamente) que disponen, además, de un portal temático dedicado al patrimonio.

4.

\section{Discusión y conclusiones}

Los resultados anteriormente reportados nos permiten responder, de forma más o menos certera, a los objetivos propuestos al comienzo del trabajo. En primer lugar, los elementos en los cuales recae el mayor protagonismo de la narrativa o discurso patrimonial autorizado de los concejos son, en lo material, las edificaciones religiosas - a saber: iglesias, capillas, ermitas o monasterios - y, en lo inmaterial, los eventos festivos municipales -en España- y la gastronomía típica de las localidades - en Portugal-. En cuanto a los encuadres de la información patrimonial, las "identidades proyecto" de los municipios ibéricos se apoyan en el énfasis local de sus activos culturales. De este modo, se reafirman a nivel endogrupal en el legado de sus generaciones pasadas, como reacción ante los fenómenos globalizadores de la cultura.

Asimismo, es posible afirmar que, tras el local, el enfoque prioritario de la información en España es el regional; no así en Portugal, donde prima una aproximación de perfil nacional. Esta circunstancia es congruente con la propia estructura y organización de los países, ya que España - más extenso y cuya distribución político-territorial se basa en el Estado de las Autonomías - está constituida por muchas regiones, algunas de ellas con marcadas identidades propias - los casos de Cataluña, Galicia o País Vasco podrían ser los más evidentes-; mientras que Portugal - menos extenso y de carácter centralizado - apela a su condición de nación culturalmente unificada. En todo caso, y tras las comparaciones efectuadas gracias al I2PC, la instantánea patrimonial que se ha tomado de ambos países, a nivel digital, evidencia una fisonomía muy parecida, y no únicamente entre España y Portugal, sino también entre las regiones litorales e interiores de ambos países. Este hallazgo revela la similar importancia que los municipios le conceden a su herencia patrimonial en términos turísticos e identitarios; y es que, como afirman Caldevilla et al., (2019): 
Desde el punto de vista de los países receptores de turistas aparece, a causa del fenómeno globalizador, una antes minoritaria o inexistente urgencia por conocer y apuntalar la propia cultura y dar un nuevo propósito al patrimonio. En la parte invitada - los turistas - surge igualmente una nueva vertiente sensibilizada con el cuidado de aquello que visitan, de su integridad ambiental y su valor cultural. Todo ello nos lleva al turismo cultural como agente reconstituyente de la cultura local frente al efecto globalizador. (Caldevilla et al., 2019, p. 61)

No obstante, y pese a los esfuerzos de los destinos locales en comunicar su patrimonio a través de Internet - repárese, sino, en la elevada proporción de ayuntamientos que disponen de un sitio web temáti$\mathrm{co}-\mathrm{y}$, por ende, sus atractivos turístico-culturales, la modalidad de las " $3 S$ " - del inglés: sea, sun and sand - sigue dominando el panorama turístico en el territorio ibérico. No se puede obviar que el enclave geográfico de la península es privilegiado desde un punto de vista climático y orográfico, dada la gran variedad de destinos de litoral que existen - atlánticos, cantábricos, mediterráneos, continentales o insulares-. Asimismo, la industria del turismo está tan fragmentada que, pese a la hegemonía del modelo masivo, existen también otras muchas opciones que reducen la proporción del turismo cultural y "de distinción" como, por ejemplo, el natural, el rural o el comercial. En todo caso, los destinos deben seguir apostando por el uso estratégico de la comunicación digital para divulgar sus activos y adaptarse a la máxima del "think globally, act locally"; es decir, ser conscientes de que forman parte de un entramado global cuyas dinámicas les afectan, y cuyos efectos pueden paliar en el seno de su propio dominio mediante este tipo de instrumentos.

En cuanto a las limitaciones del trabajo, cabe reconocer que la recogida de los datos en dos etapas - puesto que constituyeron dos estudios independientes - afecta a la correspondencia y comparación de los resultados entre países. Por otro lado, la dificultad de inventariar las manifestaciones del patrimonio y la fiabilidad de algunas de las variables también restan, respectivamente, capacidad descriptiva y objetividad a la investigación.

Para terminar, y aun de modo muy superficial, es imperativo aludir a conceptos como los de "sostenibilidad" (Budeanu et al., 2016; Hall et al., 2015), "gen- trificación" (González-Pérez, 2019; Gravari-Barbas \& Guinand, 2017), "overtourism" (Alonso-Almeida et al., 2019; Milano et al., 2019) o "turismofobia" (Zerva et al., 2019; Muñoz-de-Escalona, 2020). Son muy pocos quienes todavía albergan dudas al respecto de sus efectos en la dinámica cotidiana de aquellas sociedades receptoras de turismo. ¿De qué modo pueden los destinos combatir las consecuencias negativas de una industria, con todo, muy lucrativa e indispensable para su sustento económico, sin correr el riesgo de agotar los recursos ambientales, urbanos e, incluso, humanos? Es un interrogante verdaderamente difícil de responder, máxime si tenemos en cuenta el cambio de paradigma que se avecina tras la expansión del coronavirus "COVID-19", y la fuerte crisis económica asociada que hará tambalear los pilares en los que se asientan las sociedades de todo el mundo.

\section{Financiación}

Este trabajo ha sido financiado, en parte, por la "Fundação para a Ciência e a Tecnologia" de Portugal (Ref./no: SFRH/BPD/122402/2016).

\section{5.}

\section{Referencias}

Alonso-Almeida, M.-D.-M., Borrajo-Millán, F., \& Yi, L. (2019). Are Social Media Data Pushing Overtourism? The Case of Barcelona and Chinese Tourists. Sustainability, 11(2), Article 3356. https://doi.org/10.3390/su11123356

Bauman, Z. (2013). La cultura en el mundo de la modernidad líquida. México D.F.: Fondo de Cultura Económica.

Bessière, J. (1998). Local development and heritage: Traditional food and cuisine as tourist attractions in rural areas. Sociologia Ruralis, 38(1), 21-34. https://doi.org/10.1111/1467-9523.00061 
Bessière, J. (2013). 'Heritagisation', a challenge for tourism promotion and regional development: An example of food heritage. Journal of Heritage Tourism, 8(4), 275-291.

https://doi.org/10.1080/1743873X.2013.770861

Blackshaw, T. (2013). How Shopping Changed Leisure. En Blackshaw, T. (Ed.), Routledge Handbook of Leisure Studies (pp. 358-372). Abingdon: Routledge.

Bolívar, A. (2001). Globalización e identidades: (des) territorialización de la cultura. Revista de educación, 1, 265-288. https://bit.ly/3bq6uBp

Borah, P. (2011). Conceptual issues in framing theory: A systematic examination of a decade's literature. Journal of Communication, 61(2), 246263. https://bit.ly/3daybPi

Bryant, J., \& Miron, D. (2004). Theory and research in mass communication. Journal of Communication, 54(4), 662-704. https://bit.ly/3fuEIWz

Budeanu, A., Miller, G., Moscardo, G., \& Ooi, C. S. (2016). Sustainable tourism, progress, challenges and opportunities: an introduction. Journal of Cleaner Production, 111, 285-294. https://doi.org/10.1016/j.jclepro.2015.10.027

Caldevilla, D., García, E., \& Barrientos, A. (2019). La importancia del turismo cultural como medio de dignificación del turista y de la industria. Mediaciones Sociales, 18, 59-69. https://doi.org/10.5209/meso.65117

Capriotti, P. (2008). La planificación estratégica de la comunicación del patrimonio cultural. En Mateos, S. M. (Ed.), La comunicación global del patrimonio cultural (pp. 133-154). Gijón: Ediciones Trea.

Castells, M. (2006a). Globalisation and identity. A comparative perspective. Transfer: journal of contemporary culture, 1, 56-67.

https://bit.ly/39kXoWz
Castells, M. (2006b). Informacionalismo, redes y sociedad red: una propuesta teórica. En Castells, M. (Ed.), La Sociedad Red: una visión global (pp. 27-75). Madrid: Alianza Editorial.

Cohen, E. (2005). Principales tendencias en el turismo contemporáneo. Política y Sociedad, 42(1), 11-24. https://bit.ly/2UEzxKQ

Denzin, N. K. (2012). Triangulation 2.0. Journal of Mixed Methods Research, 6(2), 80-88. https://doi.org/10.1177/1558689812437186

Entman, R. M. (1993). Framing: toward clarification of a fractured paradigm. Journal of Communication, 43(4), 51-58. https://bit.ly/3hraup4

Featherstone, M. (1990). Global culture: An introduction. Theory, Culture \& Society, 7(2-3), 1-14. https://bit.ly/3amFuTi

Galí-Espelt, N. (2012). Identifying cultural tourism: a theoretical methodological proposal. Journal of Heritage Tourism, 7(1), 45-58.

https://doi.org/10.1080/1743873X.2011.632480

González-Pérez, J. M. (2019). The dispute over tourist cities. Tourism gentrification in the historic Centre of Palma (Majorca, Spain). Tourism Geographies, 22(1), 171-191.

https://doi.org/10.1080/14616688.2019.1586986

Gravari-Barbas, M., \& Guinand, S. (Eds.) (2017). Tourism and gentrification in contemporary metropolises: International perspectives. London \& New York: Routledge.

Hair, J. F., Black, W. C., Babin, B. J., \& Anderson, R. E. (2010). Multivariate data analysis (7th Ed.). Upper Saddle River, New Jersey: Pearson Prentice Hall.

Hall, C. M., Gossling, S., \& Scott, D. (Eds.) (2015). The Routledge handbook of tourism and sustainability. London \& New York: Routledge.

Hallett, R. W., \& Kaplan-Weinger, J. (2010). Official Tourism Websites: A Discourse Analysis Perspective. Bristol: Channel View Publications. 
Hanitzsch, T. (2007). Deconstructing Journalism Culture: Toward a Universal Theory. Communication Theory, 17(4), 367-385.

https://bit.ly/3hoZzwb

Hayes, A. F., \& Krippendorff, K. (2007). Answering the call for a standard reliability measure for $\mathrm{Co}^{-}$ ding data. Communication Methods and Measures, 1(1), 77-89.

https://doi.org/10.1080/19312450709336664

Hofstede, G., Hofstede, G. J., \& Minkov, M. (2010). Cultures and Organizations. Software of the Mind. Intercultural Cooperation and Its Importance for Survival (3rd ed.). New York: McGraw Hill Professional.

ICOMOS (1999). El buen uso del patrimonio. México: ICOMOS.

https://bit.ly/2QMGici

Igartua, J. J. (2006). Métodos cuantitativos de investigación en comunicación. Barcelona: Bosch.

Krippendorff, K. (2011). Agreement and information in the reliability of coding. Communication $\mathrm{Me}-$ thods and Measures, 5(2), 93-112. https://doi.org/10.1080/19312458.2011.568376

Lowenthal, D. (2005). Natural and Cultural Heritage. International Journal of Heritage Studies, 11(1), 81-92. https://doi.org/10.1080/13527250500037088

Matthes, J. (2009). What's in a frame? A content analysis of media framing studies in the world's leading communication journals, 1990-2005. Journalism and Mass Communication Quarterly, 86(2), 349-367.

https://doi.org/10.1177/107769900908600206

Milano, C., Novelli, M., \& Cheer, J. M. (2019). Overtourism and tourismphobia: A journey through four decades of tourism development, planning and local concerns. Tourism Planning \& Development, 16(4), 353-357. https://doi.org/10.1080/21568316.2019.1599604
Muñoz-de-Escalona, F. (2020). Un efecto imprevisto por la actividad y la doctrina turística: la turismofobia. Revista Turismo: Estudos \& Práticas, 9(1), 1-21. https://bit.ly/3frRhTd

Neuendorf, K. A. (2017). The content analysis guidebook (2nd Ed.). Thousand Oaks, California: Sage.

Organisation for Economic Co-operation and Development (2009). The impact of culture on tourism. Paris: OECD Publications.

https://bit.ly/2wCCEL6

Piñeiro-Naval, V., \& Igartua, J. J. (2012). La difusión del patrimonio a través de Internet. El caso de Castilla y León. Cuadernos de Turismo, 30, 191217. http://bit.ly/2molBNF

Piñeiro-Naval, V., Igartua, J. J., Marañón-Lazcano, F. J., \& Sánchez-Nuevo, L. A. (2018). El análisis de contenido y su aplicación a entornos web: un caso empírico. En Caffarel, C., Gaitán, J. A., Lozano, C., \& Piñuel, J. L. (Eds.), Tendencias metodológicas en la investigación académica sobre Comunicación (pp. 253-272). Salamanca: Comunicación Social.

Piñeiro-Naval, V., Igartua, J. J., \& Rodríguez-de-Dios, I. (2018). Identity- related implications of the dissemination of cultural heritage through the Internet: A study based on Framing Theory. Communication \& Society, 31(1), 1-21. https://bit.ly/3bwnnuq

Piñeiro-Naval, V., \& Mangana, R. (2018). Teoría del encuadre: Panorámica conceptual y estado del arte en el contexto hispano. Estudios sobre el Mensaje Periodístico, 24(2), 1541-1557. https://doi.org/10.5209/ESMP.62233

Piñeiro-Naval, V., \& Mangana, R. (2019). La presencia del framing en los artículos publicados en revistas hispanoamericanas de comunicación indexadas en Scopus. Palabra Clave, 22(1), e2216. https://doi.org/10.5294/pacla.2019.22.1.6 
Piñeiro-Naval, V., \& Morais, R. (2020). Cultura, patrimonio y turismo como campos de estudio para la comunicación: una revisión sistemática de la literatura hispánica reciente. Obra Digital, 18, 33-46.

https://doi.org/10.25029/od.2020.252.18

Piñeiro-Naval, V., \& Serra, P. (2019). How do destinations frame cultural heritage? Content analysis of Portugal's municipal websites. Sustainability, 11(4), Article 947.

https://doi.org/10.3390/su11040947

Richards, G. (2014). Cultural tourism 3.0: The future of urban tourism in Europe? En Garibaldi, R. (Ed.), Il turismo culturale europeo. Città ri-visitate. Nuove idee e forme del turismo culturale (pp. 2538). Milano: Franco Angeli Edizioni.

Richards, G., \& Marques, L. (2018). Creating synergies between cultural policy and tourism for permanent and temporary citizens. Barcelona: Institute of Culture of the City of Barcelona (ICUB). https://bit.ly/39rDQi5

Ritzer, G. (1992). The McDonaldization of society. Newbury Park, California: Pine Forge Press.

Robertson, R. (2000). Globalización: tiempo-espacio y homogeneidad-heterogeneidad. Zona Abierta, 92-93, 213-242. https://bit.ly/3brZ5Bv

Sacco, P. L. (2011). Culture 3.o. A new perspective for the EU 2014-2020 structural funds programming. https://bit.ly/2JfQkhV

Saperas, E., \& Carrasco-Campos, Á. (2015). La operacionalización de la noción de 'framing' en 'Journal of Communication' (2009-2013): objetos de estudio, técnicas de investigación y construcción teórica. Communication \& Society, 28(4), 49-66.

https://doi.org/10.15581/003.28.4.sp.49-66
Scheufele, D. A., \& Tewksbury, D. (2007). Framing, agenda-setting, and priming: The evolution of three media effects models. Journal of Communication, 57(1), 9-20.

https://doi.org/10.1111/j.0021-9916.2007.00326.x

Smith, L. (2006). Uses of heritage. New York: Routledge.

Tajfel, H. (1981). Human Groups and Social Categories. Cambridge: Cambridge University Press.

UNESCO (2003). Convención para la salvaguarda del Patrimonio Cultural Inmaterial. $32^{\mathrm{a}}$ reunión de la UNESCO. París, Francia.

https://bit.ly/3bpAai7

UNESCO (2005). Directrices prácticas para la aplicación de la Convención del Patrimonio Mundial. París: Centro del Patrimonio Mundial de la UNESCO. https://bit.ly/2JjDzCW

Urry, J. (1999). Globalization and citizenship. Journal of world-Systems Research, 5(2), 310-324. https://doi.org/10.5195/jwsr.1999.137

Vicente-Mariño, M., \& López-Rabadán, P. (2009). Resultados actuales de la investigación sobre framing: sólido avance internacional y arranque de la especialidad en España. Zer. Revista de Estudios de Comunicación, 14(26), 13-34. https://bit.ly/3dwjxDm

Vreese, C. H. de (2005). News framing: Theory and typology. Information Design Journal, 13(1), 5162. https://doi.org/10.1075/idjdd.13.1.06vre

Waterton, E., \& Smith, L. (2010). The recognition and misrecognition of community heritage. International Journal of Heritage Studies, 16(1-2), 4-15.

https://doi.org/10.1080/13527250903441671

White, M.D., \& Marsh, E.E. (2006). Content Analysis: A Flexible Methodology. Library Trends, 55(1), 22-45. https://doi.org/10.1353/lib.2006.0053 
Wimmer, R. D., \& Dominick, J. R. (2011). Mass Media Research: An Introduction (9th Ed.). Boston: Wadsworth.

World Tourism Organization (2018). Tourism and culture synergies. Madrid: UNWTO.

https://doi.org/10.18111/9789284418978

Zerva, K., Palou, S., Blasco, D., \& Donaire, J. A. B. (2019). Tourism-philia versus tourism-phobia: residents and destination management organization's publicly expressed tourism perceptions in Barcelona. Tourism Geographies, 21(2), 306329.

https://doi.org/10.1080/14616688.2018.1522510

Zhao, X., Liu, J. S., \& Deng, K. (2013). Assumptions behind Intercoder Reliability Indices. Annals of the International Communication Association, 36(1), 419-480.

https://doi.org/10.1080/23808985.2013.11679142 